\title{
Wideband Directional Coupler for Millimeter Wave Application based on Substrate Integrated Waveguide
}

\author{
Mohammad Javad Tavakoli ${ }^{a^{*}}$, Ali Reza Mallahzadeh ${ }^{\text {a }}$ \\ ${ }^{a}$ Electrical Engineering Department, Shahed University, Tehran, Iran
}

\begin{abstract}
Recently, Substrate Integrated Waveguide (SIW) techniques have been noticed for millimeter wave devices in microwave applications. In this paper, we are developing a wide band directional $3 \mathrm{~dB}$ coupler with a phase of $90^{\circ}$ phase delay in the range of $30-40 \mathrm{GHz}$ based on periodic vias and multi hole structure. For achieving this wide bandwidth multi-section coupler is designed based on the theoretical modeling and the simulation result is compared with HFSS and CST with two different numerical methods show good performance with low insertion and return loss, broad operational bandwidth and high isolation. A fractional bandwidth is about $28.5 \%$.
\end{abstract}

Keywords:

Substrate Integrated Waveguide;

Coupler;

Millimeter wave.

\section{Article History:}

Received: 21 September 2017

Accepted: $\quad 05$ March 2018

\section{1- Introduction}

Recently, Millimeter wave ( $\mathrm{mm}$ ) range has been noticed for its compact size of the devices and less microwave loss in range of Extremely high frequency (EHF) in the electromagnetic spectrum from 30 to 300 gigahertz and the band of $38.6-40.0 \mathrm{GHz}$ is implemented for licensed high-speed microwave data links at USA region [1]. In the mm-wave regime, the compromise is often between accurate and expensive CNC or EDM machining of waveguide technology on one hand, and inexpensive but higher-loss SIW circuitry on the other. Moreover, for broadband mm-wave applications, it is often difficult to satisfy all-metal waveguide design specifications as dimensional parameters become too small even for advanced fabrication techniques [2]. Therefore, the SIW is attractive for the extension of microwave and millimeter wave applications such as cavity filters [3], antennas [4], phase shifter [5], power dividers [6] and Diplexers [7].

In the other hand, the substrate integrated waveguide is suggested at high frequency applications and exactly it is working as same as rectangular waveguide that the sidewalls are replaced by two rows of metallic posts [8]. Therefore, The Substrate Integrated Waveguide technology usually has been used in mm-wave systems and has advantages such as high Q-factor, compact profile, low cost platform and easy fabrication [9].

Directional $3 \mathrm{~dB}$ coupler or $90^{\circ}$ degree hybrids have been implemented in various applications in communication circuits such as modulators, mixers, feed networks and other microwave devices. Branch line, Lange, Bethe hole, short slot and cruciform coupler are well-known conventional types of 90-degree hybrids [10].

In the last decade, various form of the SIW coupler are suggested for radar or mm-wave application for $90^{\circ}$ phase delay such as sum-difference comparator [11], HMSIW with double-slot coupler [12], cruciform directional coupler [13] crossed-SIW directional couplers with different angles [14] and Waveguide-Hybrid Branch Line Coupler [15]. They are also noticed for $180^{\circ}$ phase delay such as Narrow wall directional coupler [16] Half Mode Substrate Integrated Waveguide (HMSIW) for $180^{\circ}$ phase delay [17], non-uniform width [18]. Furthermore, multi-layer SIW structures are attractive for directional coupler based on coupling from Bethe hole [19-20]. Apparently, in single layer SIW the Bethe hole made between two waveguide by the elimination of some vias.

This paper presents $3 \mathrm{~dB}$ H-plane SIW coupler designs for the 30-40 GHz frequency range. In order to meet the

\footnotetext{
* CONTACT: Mj.tavakoli@shahed.ac.ir

DOI: http://dx.doi.org/10.28991/esj-2018-01132

(C) This is an open access article under the CC-BY license (https://creativecommons.org/licenses/by/4.0/).
} 
challenging specifications in terms of both operating frequency and bandwidth, new multi-branch SIW H-plane couplers on a single-layer substrate are proposed. The bandwidth around $28.5 \%$ is achieved and at last, the results are compared with previous research.

\section{2- Design Theory}

The $\mathrm{TE}_{10}$ is known as dominate mode in the waveguide and cut off frequency define as shows in Equation 1; where $a$ and $b$ are selected for width and height of the conventional waveguide. $c$ is the light speed and $\varepsilon_{r}$ is the substrate permittivity [21]. The distance between the via (S) is affected on the radiation losses will create because of the leakage field in SIW structure and $S \leq 2 d$ and $d \leq \lambda_{\mathrm{g}} / 5$ are given ideal situation where the $d$ is the diameter of the via. The guided wavelength for the dominant mode in SIW is obtained by Equation 2 [22]:

$$
\begin{aligned}
& a=\frac{c}{2 f_{c 10} \sqrt{\varepsilon_{r}}} \\
& \lambda_{g 10}=\frac{2 \pi}{\sqrt{\left(\frac{\varepsilon_{r} \omega}{c}\right)^{2}+\left(\frac{\pi}{a}\right)^{2}}}
\end{aligned}
$$

Multi-hole coupling structures are noticeably for enhancement of the bandwidth and coupling characteristic in directional coupler with various techniques such binomial, Chebyshev, and superimposed to calculate the coupling of each hole. In addition, many researches have been done around the effect of the number of holes on directivity and them shows that distances between the holes and hole dimensions will effect on the coupler characteristic [23]. The multihole waveguide coupler is employed in this design, and as shows in Figure 1 they are a few holes with uniform arrangement are modified here for the conventional directional coupler. This method is a useful technique for improving the bandwidth of the coupler.

However, the best dimension of the hole is obtained for diameter of $\lambda_{\mathrm{g}} / 4$ and for wideband structure the $\lambda_{\mathrm{g}}$ is obtained by Equation 3 where $\lambda_{\mathrm{g} 1}$ and $\lambda_{\mathrm{g} 2}$ are for upper and lower frequency:

$$
\lambda_{g}=\frac{\lambda_{g 1}+\lambda_{g 2}}{2}
$$

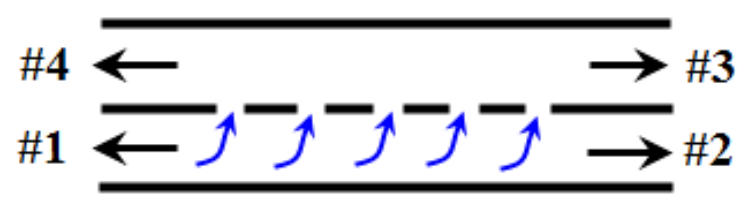

Figure 1. Multi-hole coupling structure.

The even and odd modes or mode matching method can be used for modeling the SIW coupler [24-25]. However, for common design, the length of each branch is assumed $\lambda_{\mathrm{g}} / 2$ and the width of the aperture is $\lambda_{\mathrm{g}}[16]$.

\section{3- The Coupler Design}

Figure 2 (a) shows the basic model of the $3 \mathrm{~dB}$ SIW coupler with Symmetrical dimensions [26]. Here, $\mathrm{TE}_{10}$ is the dominate mode so each branch of the coupler is working as a conventional rectangular waveguide and widest aperture is modified for coupling between top and bottom waveguide and the step shape of the waveguide at center part is used for matching. The frequency band of the waveguide determines the size of the waveguide by Equation 1 . The diameter of the via is chosen to be equal or smaller than a tenth of the wavelength of the maximum operating frequency for less linkage loss.

Figure 2 (b) shows the geometry of the prototype directional coupler for MM wave application.

The taper microstrip line is used for matching connecting of the SIW part to50 $\Omega$ feed with bent line. It is a common method for wideband SIW line [27-28]. Thus, the width and length are chosen so that the input impedance of the microstrip feed line matched to the SIW input impedance. Figure 2 (c) shows the geometry of the prototype feed line with taper formation.

A prototype $3 \mathrm{~dB}$ SIW symmetrical coupler is designed by replacing the single hole by the multi section structures. Figure 2 (b) shows the designed $3 \mathrm{~dB}$ coupler geometrical dimension for 30-40 GHz. The coupler is designed on a Rogers RT/Duroid 5880 substrate. The height of the substrate is $0.508 \mathrm{~mm}$ with (loss tangent 0.0009 ) with total dimension of $38.973 \times 93.33 \mathrm{~mm}^{2}$. In addition, all dimensions have been presented in Table 1 .

As described in pervious part, we have two essential parts in our design. At first based on the dominate mode, the dimensions of the waveguide are calculated and assumed the initial value of the $\lambda_{\mathrm{g}}$ of the center frequency. In the second 
part, we should modify the structure and connect it to SMA connector with matching taper line.

In the E-plane branch-line coupler, the branch parts are between the broad walls of the main waveguides so there is a plane of symmetry through the centers of the broad walls of all the waveguides.

It reduces the aperture sizes and thus increases the distance between apertures that are later to be manufactured by via holes. Finally, fine optimizations for the $3 \mathrm{~dB}$ coupler are required to ensure that all dimensions fall into the possible range of via holes. Combining the E-plane, multi-branch line coupler and the main directional coupler that is introduced above, lead to wideband directional coupler.

Table 1. The geometry of the prototype coupler.

\begin{tabular}{ccc}
\hline parameter & mm & Wavelength \\
\hline $\mathrm{L}_{1}$ & 7.7 & $1.14 \lambda_{\mathrm{g} 0}$ \\
$\mathrm{~L}_{2}$ & 14.6 & $2.16 \lambda_{\mathrm{g} 0}$ \\
$\mathrm{~L}_{3}$ & 30.48 & $4.68 \lambda_{\mathrm{g} 0}$ \\
$\mathrm{~L}_{4}$ & 2.06 & $0.3 \lambda_{\mathrm{g} 0}$ \\
$\mathrm{~L}_{5}$ & 4 & $0.6 \lambda_{\mathrm{g} 0}$ \\
$\mathrm{~L}_{6}$ & 10 & $1.48 \lambda_{\mathrm{g} 0}$ \\
$\mathrm{~L}_{7}$ & 14.8 & $2.19 \lambda_{\mathrm{g} 0}$ \\
$\mathrm{~W}_{1}$ & 4.6 & $0.68 \lambda_{\mathrm{g} 0}$ \\
$\mathrm{~W}_{2}$ & 3.9 & $0.57 \lambda_{\mathrm{g} 0}$ \\
$\mathrm{~W}_{3}$ & 16.452 & $2.43 \lambda_{\mathrm{g} 0}$ \\
$\mathrm{~W}_{4}$ & 3 & $0.44 \lambda_{\mathrm{g} 0}$ \\
$\mathrm{~W}_{5}$ & 1.2 & $0.17 \lambda_{\mathrm{g} 0}$ \\
\hline
\end{tabular}

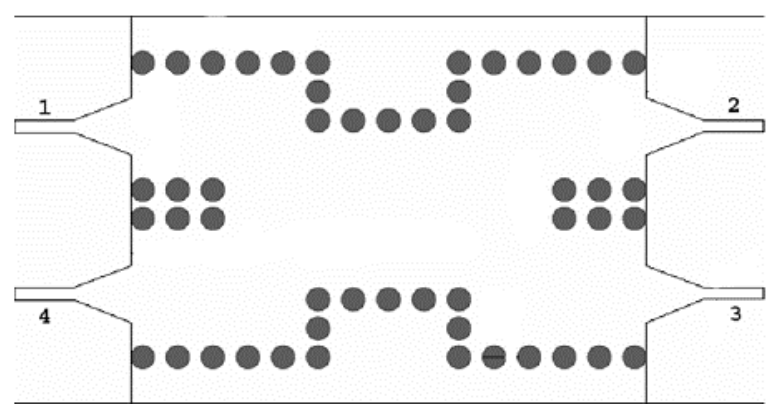

(a)

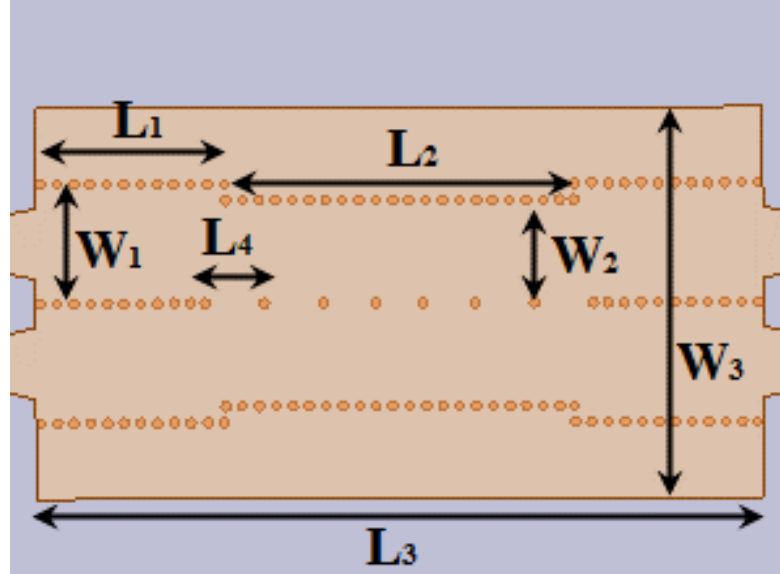

(b)

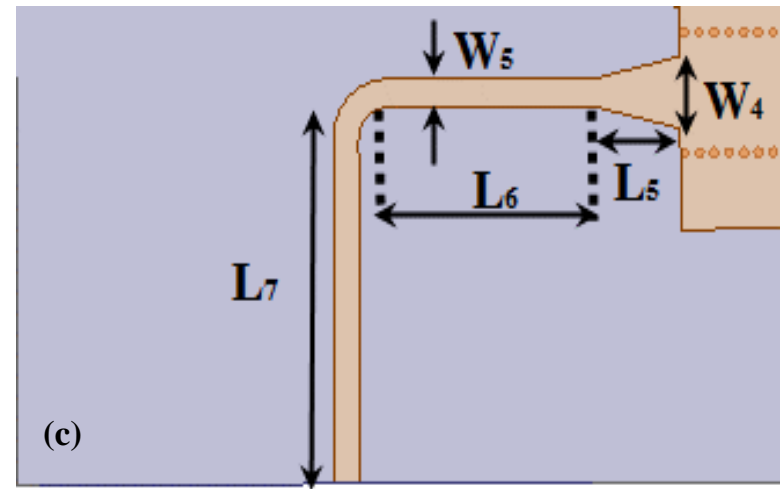

Figure 2. Geometry of the coupler (a) primary SIW coupler (b) the prototype SIW coupler (c) the final prototype coupler. 


\section{4- Simulation Results}

The design parameters tuned finely by using three-dimensional (3-D) electromagnetic (EM) simulation software high frequency structure simulator (HFSS) to achieve wide band performance. In the first step, we designed conventional branch line coupler without multi hole and then the result is compared with final directional coupler when the multi hole techniques are implemented. Figure 3 shows a comparison between the suggested model of coupler in the presence and absence of the vias. As shows here, for $\mathrm{S}_{11}$ (reflection factor) are around $-16 \mathrm{~dB}$ in the range of $31-37 \mathrm{GHz}$ for simple model and when the vias are implemented, the result is improved and $S_{11}$ is reduced to $-40 \mathrm{~dB}$ and bandwidth enhanced and increased. In this case, the coupler is covered range of 30-40 GHz as shows in Figure 3 (a). Figure 3 (d) shows that we have similar condition for $\mathrm{S}_{14}$ (Isolation factor) and the return loss value and bandwidth is increased drastically.

For $\mathrm{S}_{12}$ (through factor) is around -3 to $-4 \mathrm{~dB}$ in the range of $30-37 \mathrm{GHz}$ for simple model and when the vias are implemented, the result is improved and the bandwidth enhanced and increased. In this case, the coupler is covered range of 30-40 GHz as shows in Figure 3 (c) with -3 to $-4 \mathrm{~dB}$ loss. Figure 3 (d) shows that we have similar condition for $\mathrm{S}_{13}$ (coupling factor) and the return loss value is around -3.8 to $-4.8 \mathrm{~dB}$ for $30-40 \mathrm{GHz}$.

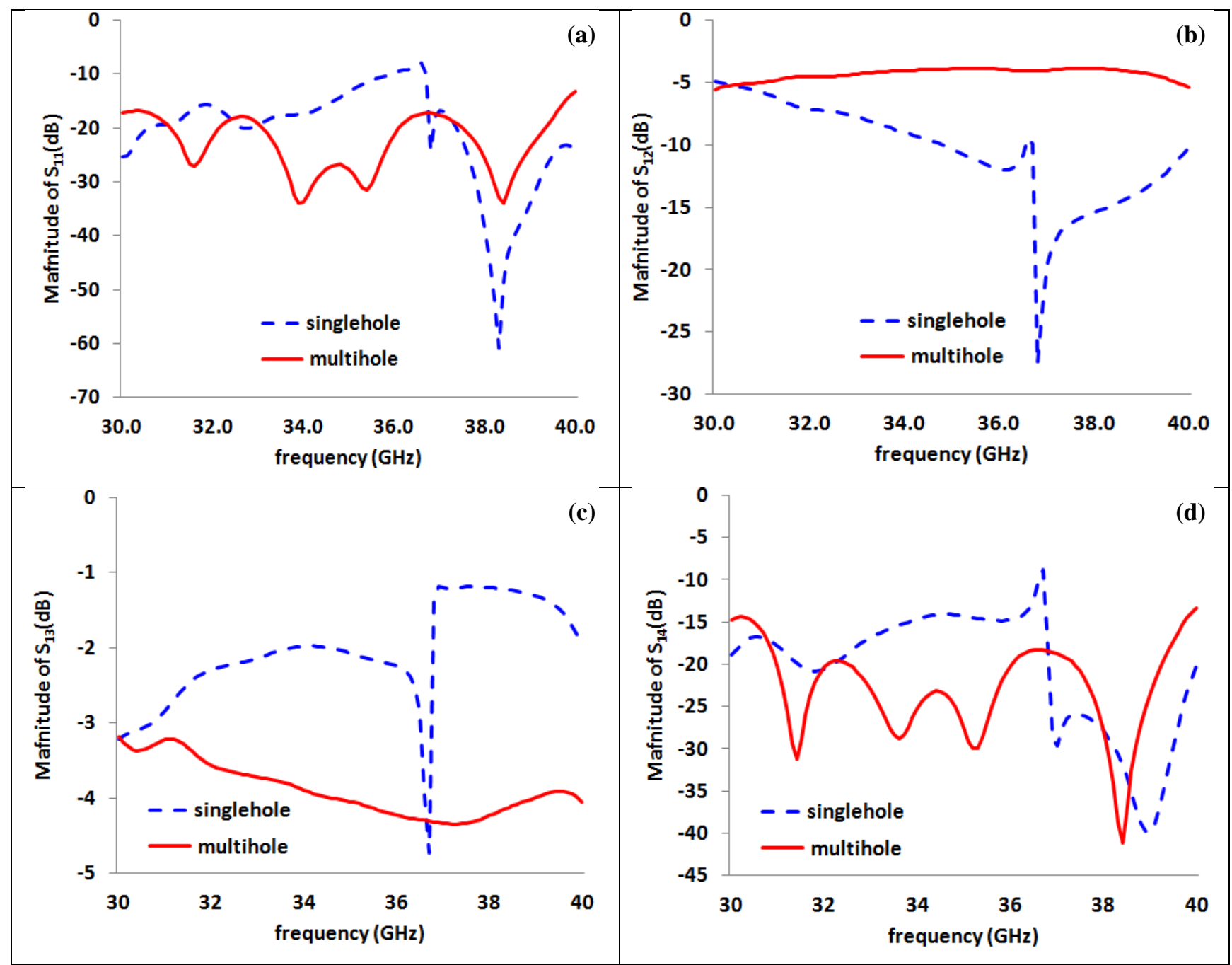

Figure 3. Comparison between suggested model of coupler in presence and absence of the vias (a)S11 or reflection factor (b) S12 or through factor (c) S13 or coupling factor (d) S14 or Isolation factor.

Figure 4 shows the phase difference between two output ports with single-hole and with multi-hole techniques. It can be seen the outputs at ports 2 and 3 are $-3.8 \mathrm{~dB}$ to $-4.5 \mathrm{~dB}$, respectively, the phase difference is distributed in the range of $88.3 \sim 92.5^{\circ}$ within the frequency band of 30 to $40 \mathrm{GHz}$. 


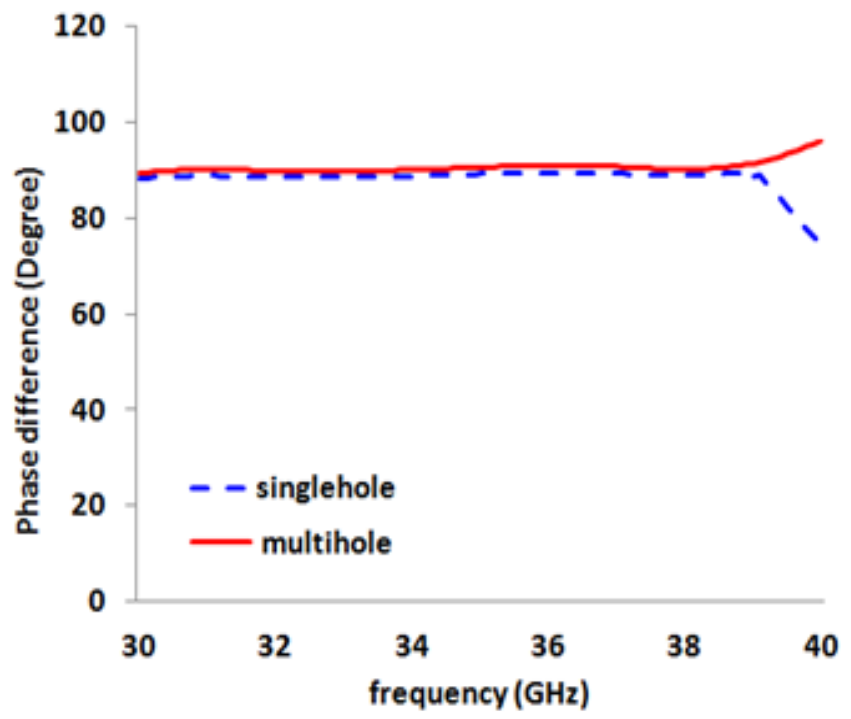

Figure 4. Simulated phase difference between output ports for both case study of the prototype coupler.

Figure 5 shows the current distribution at $35 \mathrm{GHz}$ on the surface of the prototype coupler. It is found that the input signal from port 1 interacts with metallic posts in the hole region, and is coupled equally to port 2 and port 3 . We show the current distribution for single hole and multi-hole method at Figure 5 (a) and (b) respectively.
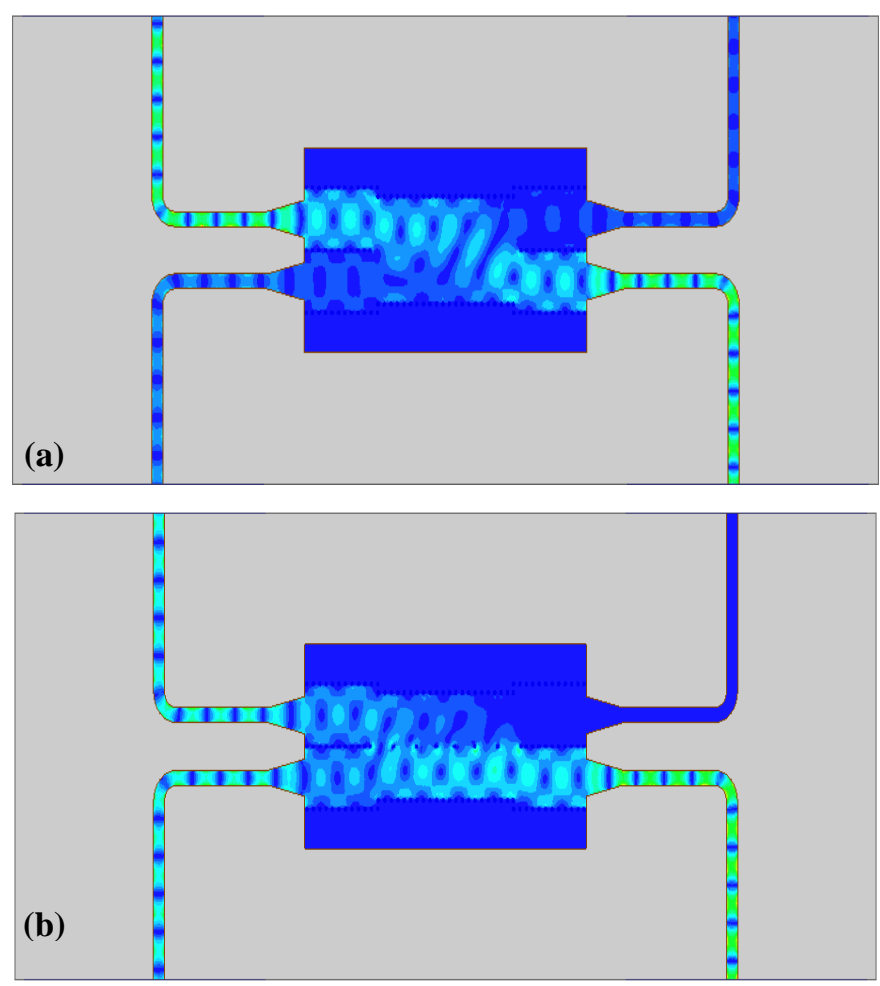

Figure 5. Simulated current distribution at $35 \mathrm{GHz}$ for (a) single hole model (b) multi hole model.

\section{5- Comparison}

This structure is noticed in many researches of the symmetrical form and reciprocal response [13] however asymmetric forms are studied in pervious researches [25]. In the other multi- hole are noticed for improving the resulting bandwidth [24, 29]. In this section, we presented a comparison between current work and pervious models of the SIW coupler and the comparison is given at Table 2.

We have noticed bandwidth and $S_{12}$ as important values and on the other hand, compared phase and length of them in wavelength. In addition, we are noticing the structure as a symmetric or asymmetric structure and denoted them by (S) and (A) in Table 2. 
Table2. Comparison of the prototype coupler.

\begin{tabular}{cccc}
\hline Reference & Bandwidth (\%) & Phase/Type & $\mathbf{S}_{\mathbf{1 2}}$ \\
\hline Present model & $28.5 \%$ & $90 \% \mathrm{~S}$ & -2.5 to $-4.5 \mathrm{~dB}$ \\
{$[10]$} & $35 \%$ & $90 \% / \mathrm{S}$ & -4 to $-5.5 \mathrm{~dB}$ \\
{$[11]$} & $13 \%$ & $90 \% \mathrm{~S}$ & -2.5 to $-4 \mathrm{~dB}$ \\
{$[12]$} & $14.2 \%$ & $90 \% / \mathrm{S}$ & -4 to $-4.5 \mathrm{~dB}$ \\
{$[13]$} & $18 \%$ & $90 \% / \mathrm{S}$ & -3 to $-4 \mathrm{~dB}$ \\
{$[14]$} & $22 \%$ & $90 \% \mathrm{~S}$ & -3 to $-5 \mathrm{~dB}$ \\
{$[15]$} & $1.7 \%$ & $90 \% \mathrm{~S}$ & -3 to $-5 \mathrm{~dB}$ \\
{$[16]$} & $5.4 \%$ & $180 \% \mathrm{~S}$ & -3.7 to $-4.5 \mathrm{~dB}$ \\
{$[17]$} & $19 \%$ & $180 \% \mathrm{~A}$ & -3 to $-5 \mathrm{~dB}$ \\
{$[25]$} & $25 \%$ & $90 / \mathrm{A}$ & -4 to $-5 \mathrm{~dB}$ \\
\hline
\end{tabular}

\section{6- Conclusion}

Broadband directional coupler in SIW technology is presented for MM wave-band applications. The specifications towards tight coupling and broadband performance require several stages in the design process. In order to overcome higher-order mode excitation commonly observed in the upper frequency range of components with tight coupling, the design process is done to place them out of operational range. The performance of the proposed coupler for $3 \mathrm{~dB}$ demonstrates good agreement between HFSS and CST simulations. However, the results for the through and coupled ports show additional losses between $2.5 \mathrm{~dB}$ and $1.3 \mathrm{~dB}$. Although these are good results in the $30 \mathrm{GHz}$ frequency range, the main contributions to loss appear to because by the microstrip-to-SIW transition and the dielectric substrate.

\section{2- References}

[1] Junfeng Xu, Wei Hong, Hongjun Tang, Zhenqi Kuai, and Ke Wu. "Half-Mode Substrate Integrated Waveguide (HMSIW) Leaky-Wave Antenna for Millimeter-Wave Applications.” IEEE Antennas and Wireless Propagation Letters 7 (2008): 85-88. doi:10.1109/lawp.2008.919353.

[2] Claude, Stéphane. "Sideband-Separating SIS Mixer for ALMA Band 7-275-370 GHz." In Proc. 14th Int'1. Symp. on Space Terahertz Tech, pp. 22-24. 2003.

[3] Wei Shen, Xiao-Wei Sun, Wen-Yan Yin, Jun-Fa Mao, and Qi-Fu Wei. “A Novel Single-Cavity Dual Mode Substrate Integrated Waveguide Filter with Non-Resonating Node.” IEEE Microwave and Wireless Components Letters 19, no. 6 (June 2009): 368370. doi:10.1109/lmwc.2009.2020017.

[4] Zarrabi, Ferdows B., Navid P. Gandji, Rahele Ahmadian, Hamed Kuhestani, and Zahra Mansouri. "Modification of Vivaldi Antenna for 2-18 GHz UWB Application with Substrate Integration Waveguide Structure and Comb Slots." Applied Computational Electromagnetics Society Journal 30, no. 8 (2015).

[5] Kuhestani, Hamed, Mohammad Naser-Moghadasi, Mahshid Maleki, and Ferdows B. Zarrabi. "Phase Shifter Designing Base on Half Mode Substrate Integrated Waveguide with Reconfigurable Quality.” Microwave and Optical Technology Letters 57, no. 11 (August 28, 2015): 2562-2567. doi:10.1002/mop.29399.

[6] Chen, S.Y., D.S. Zhang, and Y.T. Yu. "Wideband SIW Power Divider with Improved Out-of-Band Rejection." Electronics Letters 49, no. 15 (July 18, 2013): 943-944. doi:10.1049/el.2013.0979.

[7] Tang, Hong Jun, Wei Hong, Ji-Xin Chen, Guo Qing Luo, and Ke Wu. "Development of Millimeter-Wave Planar Diplexers Based on Complementary Characters of Dual-Mode Substrate Integrated Waveguide Filters With Circular and Elliptic Cavities." IEEE Transactions on Microwave Theory and Techniques 55, no. 4 (April 2007): 776-782. doi:10.1109/tmtt.2007.893655.

[8] Mansouri, Z., M. Kishihara, F. B. Zarrabi, F. Geran, and Tehran Hesarak. "Broadband half mode substrate integrated waveguide cruciform coupler." In The Second Iranian Conference on Engineering Electromagnetics (ICEEM 2014), pp. 595-599. 2014.

[9] Kumari, Vandana, Wriddhi Bhowmik, and Shweta Srivastava. "Design of High-Gain SIW and HMSIW H-Plane Horn Antenna Using Metamaterial.” International Journal of Microwave and Wireless Technologies 7, no. 06 (July 15, 2014): 713-720. doi:10.1017/s1759078714000920.

[10] Mansouri, Zahra, Mitsuyoshi Kishihara, Ferdows B. Zarrabi, and Fatemeh Geran. "Modified Broadband Half Mode Substrate Integrated Waveguide Cruciform Coupler." Applied Computational Electromagnetics Society Journal 29, no. 11 (2014).

[11] Tang, Yiming, and Wen Wu. "Design of Substrate Integrated Waveguide Sum-Difference Comparator." 2006 7th International Symposium on Antennas, Propagation \& EM Theory (October 2006). doi:10.1109/isape.2006.353460. 
[12] Liu, B., W. Hong, Y. Zhang, J.X. Chen, and K. Wu. "Half-Mode Substrate Integrated Waveguide (HMSIW) Double-Slot Coupler." Electronics Letters 43, no. 2 (2007): 113. doi:10.1049/el:20073127.

[13] Djerafi, T., and Ke Wu. “Super-Compact Substrate Integrated Waveguide Cruciform Directional Coupler.” IEEE Microwave and Wireless Components Letters 17, no. 11 (November 2007): 757-759. doi:10.1109/lmwc.2007.908040.

[14] Yitao Wang, Xiaowei Zhu, and Ling Tian. "Design of Crossed-SIW Directional Couplers with Different Angles.” 2008 AsiaPacific Microwave Conference (December 2008). doi:10.1109/apmc.2008.4958028.

[15] Abdel-Wahab, W. M., and Safieddin Safavi-Naeini. "Low Loss Double-Layer Substrate Integrated Waveguide-Hybrid Branch Line Coupler for Mm-Wave Antenna Arrays.” 2011 IEEE International Symposium on Antennas and Propagation (APSURSI) (July 2011). doi:10.1109/aps.2011.5996917.

[16] Bing Liu, Wei Hong, ZhangCheng Hao, and Ke Wu. "Substrate Integrated Waveguide 180-Degree Narrow-Wall Directional Coupler.” 2005 Asia-Pacific Microwave Conference Proceedings (n.d.). doi:10.1109/apmc.2005.1606319.

[17] Bing Liu, Wei Hong, Yan Zhang, Hong Jun Tang, Xiaoxin Yin, and Ke Wu. "Half Mode Substrate Integrated Waveguide $180^{\circ}$ 3-dB Directional Couplers." IEEE Transactions on Microwave Theory and Techniques 55, no. 12 (December 2007): $2586-2592$. doi:10.1109/tmtt.2007.909749.

[18] Cheng, Y.J., K. Wu, and W. Hong. "Millimetre-Wave Monopulse Antenna Incorporating Substrate Integrated Waveguide Phase Shifter.” IET Microwaves, Antennas \& Propagation 2, no. 1 (February 1, 2008): 48-52. doi:10.1049/iet-map:20070134.

[19] Amine, Ali, Larbi Talbi, and Kheireddine Sellal. "Design of a Bethe-Hole Directional Coupler Using Substrate Integrated Waveguide Technique." Microwave and Optical Technology Letters 53, no. 8 (May 17, 2011): 1730-1734. doi:10.1002/mop.26119.

[20] Mbaye, Moustapha, Larbi Talbi, Khelifa Hettak, and Ali Kabiri. "Design of 15 dB Directional Coupler Using SubstrateIntegrated Waveguide Technology." Microwave and Optical Technology Letters 54, no. 4 (February 16, 2012): $970-973$. doi:10.1002/mop.26678.

[21] Rayas-Sanchez, Jose E., and Vladimir Gutierrez-Ayala. “A General EM-Based Design Procedure for Single-Layer Substrate Integrated Waveguide Interconnects with Microstrip Transitions.” 2008 IEEE MTT-S International Microwave Symposium Digest (June 2008). doi:10.1109/mwsym.2008.4632999.

[22] Ke Wu, D. Desiandes, and Y. Cassivi. "The Substrate Integrated Circuits - a New Concept for High-Frequency Electronics and Optoelectronics." 6th International Conference on Telecommunications in Modern Satellite, Cable and Broadcasting Service, 2003. TELSIKS 2003. (n.d.). doi:10.1109/telsks.2003.1246173.

[23] Moghavvemi, Mahmoud, Hossein Ameri, and Farhang Alijani. "Multi-Hole Waveguide Directional Couplers." Solutions and Applications of Scattering, Propagation, Radiation and Emission of Electromagnetic Waves (November 14, 2012). doi: $10.5772 / 51355$.

[24] Kordiboroujeni, Zamzam, Jens Bornemann, and Thomas Sieverding. "Mode-Matching Design of Substrate-Integrated Waveguide Couplers." 2012 Asia-Pacific Symposium on Electromagnetic Compatibility (May 2012). doi:10.1109/apemc.2012.6237805.

[25] Han, Liang, Ke Wu, Xiao-Ping Chen, and Fanfan He. "Accurate and Efficient Design Technique for Wideband Substrate Integrated Waveguide Directional Couplers.” International Journal of RF and Microwave Computer-Aided Engineering 22, no. 2 (December 30, 2011): 248-259. doi:10.1002/mmce.20599.

[26] Ji-Xin Chen, Wei Hong, Zhang-Cheng Hao, Hao Li, and Ke Wu. "Development of a Low Cost Microwave Mixer Using a BroadBand Substrate Integrated Waveguide (SIW) Coupler.” IEEE Microwave and Wireless Components Letters 16, no. 2 (February 2006): 84-86. doi:10.1109/lmwc.2005.863199.

[27] Bozzi, M., A. Georgiadis, and K. Wu. "Review of Substrate-Integrated Waveguide Circuits and Antennas." IET Microwaves, Antennas \& Propagation 5, no. 8 (2011): 909. doi:10.1049/iet-map.2010.0463.

[28] Deslandes, Dominic. "Design Equations for Tapered Microstrip-to-Substrate Integrated Waveguide Transitions.” 2010 IEEE MTT-S International Microwave Symposium (May 2010). doi:10.1109/mwsym.2010.5517884.

[29] Dousset, David, Jens Bornemann, Maxime Daigle, Stephane Claude, and Ke Wu. "Broadband 100 GHz Substrate-Integrated Waveguide Couplers with Irregularly Shaped Via Holes for Higher-Order Mode Suppression." 2012 42nd European Microwave Conference (October 2012). doi:10.23919/eumc.2012.6459312. 